vegetable crops as influenced by municipal waste composts. Acta Horticulturae, v. 222, p. 51-62, 1988.

GERBER, J.M.; SWIADER, J.M.; PECK, T.R. Sewage sludge on vegetables - A mixed Blessing. Illinois Research, v. 23, p. 12-13, 1981.

GILL, H.S.; MEELU, O.P. Studies on the substitution of inorganic fertilizers with organic manure and their effect on soil fertility in ricewheat rotation. Fertilizer Research, v. 3, p. 303314, 1982.

HAMPTON, M.O.; SCHAFFER, B.; BRYAN, H.H. Nutrient concentrations growth and yield of tomato and squash in municipal solid-waste amended soil. Hortscience, v. 29, p. 785-788, 1994

HOLANDA, J.J.; MIELNICZU, J.; STAMMEL, J.G. Utilização de esterco e adubo mineral em quatro sequências de culturas em solo de encosta basáltica do Rio Grande do Sul. Revista Brasileira de Ciência do Solo, v. 6, p. 47-51, 1982.

MALAVOLTA, E.; VITTI, G. C.; OLIVEIRA, S
A. Avaliação do estado nutricional das plantas: princípios e aplicações. Piracicaba: POTAFOS, 1997. 319 p.

MAZUR, N.; VELLOSO, A.C.X.; SANTOS, G. A. Efeito do composto de resíduo urbano no $\mathrm{pH}$ e alumínio trocável em solo ácido. Revista Brasileira de Ciência do Solo, v. 7, p. 157-159, 1983. MELO, W.J.; MARQUES, M.O.; SANTIAGO, G.; CHELLI, R.A. Efeito de doses crescentes de lodo de esgoto sobre frações da matéria orgânica e CTC de um Latossolo cultivado com cana-deaçúcar. Revista Brasileira de Ciência do Solo, v. 18, p. 449-455, 1994.

MUGWIRA, L.M. Residual effects of dairy cattle manure on millet and rye forage and soil properties. Journal of Environmental Quality, v. 8, p. 251-255, 1979.

MURILLO, J.M.; HERNANDEZ, J.M.; BARROSO, M.; LOPEZ, R. Produccion frente a contaminacion en la utilizacion agricola de compostos urbanos. Anales de Edafologia y Agrobiologia, v. 48, p. 143-160, 1989.

NDAYEGAMIYE, A.; CÔTÉ, D. Effect of long- term pig slurry and solid cattle manure application on soil chemical and biological properties. Canadian Journal of Soil Science, v. 69, p. 39-47, 1989.

PAVAN, M.A. Avaliação de esterco de bovino biodigerido e curtido na fertilidade do solo e na nutrição e produção do cafeeiro. Londrina: IAPAR, 1993. 16p. (IAPAR. Boletim Técnico, 45). TRANI, P.E.; RAIJ, B. van. Hortaliças. In: RAIJ, B. van; CANTARELLA, H.; QUAGGIO, J.A.; FURLANI, A.M.C., ed. Recomendações de adubação e calagem para o Estado de São Paulo. Campinas: Instituto Agronômico de Campinas, 1996. p. 155-203. (Boletim Técnico, 100)

TRANI, P.; NAGAI, H.; PASSOS, F.A. Tomate (estaqueado). In: RAIJ, B. van; CANTARELLA, H.; QUAGGIO, J.A.; FURLANI, A.M.C., ed. Recomendações de adubação e calagem para o Estado de São Paulo. Campinas: Instituto Agronômico de Campinas, 1996. p. 184. (Boletim Técnico, 100) VIVANCOS, A.D. Fertirrigacion. Madrid: Mundi-Prensa, 1993. 217 p.

MAROUELLI, W.A.; SILVA, W.L.C. Profundidade de instalação da linha de gotejadores em tomateiro para processamento industrial. Horticultura Brasileira, Brasília, v. 20, n. 2, p. 206-210, junho 2.002 .

\title{
Profundidade de instalação da linha de gotejadores em tomateiro para processamento industrial
}

Waldir A. Marouelli; Washington L.C. Silva

Embrapa Hortaliças, C. Postal 218, 70.359-970, Brasília/DF. E-mail: waldir@cnph.embrapa.br.

\section{RESUMO}

Avaliou-se a resposta do tomateiro para processamento industrial, irrigado por gotejamento, a diferentes profundidades de instalação da linha lateral de gotejadores $(0 ; 20$ e $40 \mathrm{~cm})$. Um tratamento irrigado por aspersão foi utilizado como controle. O experimento foi conduzido na Embrapa Hortaliças, nas condições edafoclimáticas da região de cerrados do Brasil Central. Maior crescimento vegetativo de plantas foi observado nos tratamentos irrigados por aspersão e gotejamento superficial, enquanto que menor crescimento ocorreu no gotejamento a $40 \mathrm{~cm}$. A produtividade comercial do tratamento irrigado por gotejamento superficial $\left(124\right.$ t.ha $\left.^{-1}\right)$ foi $32 \%$ maior que no tratamento por gotejamento subterrâneo a $40 \mathrm{~cm}, 15 \%$ maior que no tratamento irrigado por aspersão, não diferindo $(\mathrm{p}>0,05)$ do tratamento por gotejamento a $20 \mathrm{~cm}$. Incrementos de produtividade foram devidos, principalmente, ao maior número de frutos por planta, uma vez que o estande final e a massa média de frutos não foram afetados pelos tratamentos. A incidência de frutos podres na irrigação por aspersão foi 112 e $453 \%$ maior que nos tratamentos irrigados por gotejamento superficial e subterrâneo, respectivamente.

Palavras-chave: Lycopersicon esculentum, gotejamento, sistema de irrigação, gotejo subterrâneo.
ABSTRACT

\section{Drip line placement depth for processing tomatoes crop}

A field experiment was carried out at Embrapa Hortaliças under soil and climate conditions of Central Brazil "cerrado" region, aiming to evaluate the response of drip-irrigated processing tomatoes to different drip line placement depths $(0 ; 20$ and $40 \mathrm{~cm})$. A control treatment was irrigated by sprinkle system. Larger plant vegetative growth was observed for the sprinkle and surface drip treatments, while smaller growth occurred for the $40 \mathrm{~cm}$-depth drip treatment. Marketable yield of the surface drip irrigated treatment (124 t.ha $\left.{ }^{-1}\right)$ was $32 \%$ larger than the $40 \mathrm{~cm}$-depth drip treatment, $15 \%$ larger than the sprinkle treatment, but did not differ $(p>0,05)$ from the 20 $\mathrm{cm}$-depth drip treatment. Yield increments were basically due to a larger number of fruits per plant, since final stand and mean fruit mass were not affected by treatments. Rotten fruit rate for the sprinkle treatment was 112 and $453 \%$ larger than for the treatments irrigated by surface and subsurface drip, respectively.

(Aceito para publicação em 21 de fevereiro de 2.002)

A aspersão é o principal método utilizado no Brasil para a irrigação do tomateiro para processamento industrial, ocupando mais de $90 \%$ da área irrigada. Por molhar a parte aérea das plantas, a aspersão favorece uma série de doenças, podendo provocar perdas significativas de produção e qualidade de frutos (Barbosa, 1997; Marouelli \& Silva, 2000).
Keywords: Lycopersicon esculentum, trickle, irrigation system, subsurface drip.
Estudos exploratórios realizados no Brasil por Silva \& Marouelli (1995), Silva et al. (1999) e Barbosa (1997) têm demonstrado o potencial do gotejamento para a irrigação do tomateiro industrial. 
Assim, o gotejamento, que até alguns anos atrás era tido como um sistema economicamente não indicado para o tomateiro industrial no Brasil, vem se tornando uma opção viável, ainda porém com taxa de retorno ligeiramente inferior à irrigação por pivô central (Marouelli et al., 1998). Mesmo sem dispor de informações tecnológicas suficientes alguns produtores começaram a utilizar, nos últimos anos, o gotejamento superficial como uma alternativa para aumentar a produtividade e a qualidade de frutos, economizar água e minimizar o uso de fungicidas (Marouelli \& Silva, 2000). Segundo previsões das agroindústrias, a área de tomateiro industrial irrigada por gotejamento, que em 2001 totalizou 460 ha, deve atingir cerca de 1.000 ha em 2002 (Sant'Ana, 2001¹ ${ }^{1}$.

O sistema de gotejamento subterrâneo, em comparação ao superficial, tem uma série de vantagens adicionais, como aplicar água e nutrientes diretamente na zona radicular, reduzir as perdas por evaporação, evitar danos mecânicos durante as operações de cultivo e por roedores às tubulações, proporcionar maior crescimento do sistema radicular, reduzir a umidade na superfície do solo e minimizar a incidência de doenças (Camp, 1998; Silva et al., 1999).

Vários estudos vêm sendo realizados, principalmente na Califórnia, com gotejamento subterrâneo em tomateiro industrial. May (1997) obteve produtividades de até 139 t.ha ${ }^{-1}$, enquanto Phene et al. (1992) demonstraram que valores acima de 200 t.ha ${ }^{-1}$ podem ser alcançados, desde que a irrigação e a fertirrigação sejam realizadas de forma precisa e sob regime de alta freqüência. Nas condições de cerrados do Brasil Central, Silva et al. (1999) obtiveram produtividade em torno de 100 t.ha $^{-1}$. As profundidades de instalação da linha lateral de gotejadores utilizadas por May (1996), Phene et al. (1992) e Silva et al. (1999) foram de 25; 45 e $15 \mathrm{~cm}$, respectivamente.

A profundidade ideal para a instalação da linha lateral, entretanto, depende de vários fatores, especialmente das características físicas, hídricas e químicas do solo. Assim, solos profundos, férteis e com alta condutividade hidráulica não-saturada, permitem maiores profundidades de instalação da lateral. Como regra geral, Camp (1998) sugere que a profundidade deve ser suficiente para que não haja afloramento de umidade na superfície do solo e que as operações de preparo possam ser realizadas sem causar danos às laterais, haja visto que a lateral deve permanecer instalada no campo.

A principal limitação do gotejamento subterrâneo está geralmente relacionada ao estabelecimento inicial da cultura. Por não umedecer a superfície do solo ou não permitir condições satisfatórias de umidade para a germinação de sementes ou pegamento de mudas, se faz necessário o uso de um segundo sistema de irrigação, freqüentemente por aspersão, aumentando os custos e reduzindo o retorno econômico (May, 1997; Camp, 1998). Outro problema potencial é o entupimento de gotejadores devido a entrada de partículas de solo ou de raízes nos gotejadores, o que pode ser prevenido pelo uso de ventosas, gotejadores especiais e/ou injeção de Trifluralina em pequenas concentrações (Casaño, 1998).

Avaliou-se neste estudo, nas condições edafoclimáticas da região de cerrados do Brasil Central, a produção do tomateiro para processamento industrial sob diferentes profundidades de instalação da linha lateral de gotejadores.

\section{MATERIAL E MÉTODOS}

O experimento foi conduzido no campo experimental da Embrapa Hortaliças, em Brasília, DF, em um Latossolo Vermelho-Escuro, fase cerrado e de textura argilosa, durante os meses de maio a setembro de 2000. A curva média de retenção de umidade do solo (\% vol.), na camada de 0 a $40 \mathrm{~cm}$, no intervalo de tensão entre 5 e 1500 $\mathrm{kPa}$, foi ajustada à equação de van Genuchten (1980): $\theta=23,3+17,0 /\left[1+(0,0676 \times h)^{18348}\right]^{04545}$

O delineamento experimental foi de blocos ao acaso, com seis repetições. Os tratamentos consistiram de três profundidades de instalação da linha lateral de gotejadores: superficial (TGS), $20 \mathrm{~cm}$ (TG20) e $40 \mathrm{~cm}$ (TG40). Um tratamento irrigado por aspersão (TA) foi utilizado como controle.

O transplante de mudas do híbrido Heinz 9498 foi realizado no espaçamento de $30 \mathrm{~cm}$ x $120 \mathrm{~cm}$. A parcela experimental constou de 4 linhas de plantas de $6 \mathrm{~m}$ de comprimento, tendo sido colhida uma área de $10,8 \mathrm{~m}^{2}$.

A adubação, em kg.ha-1 ${ }^{-1}$, foi realizada com 120 de N, 200 de $\mathrm{K}_{2} \mathrm{O}, 600$ de $\mathrm{P}_{2} \mathrm{O}_{5}, 100$ de $\mathrm{Ca}, 25$ de $\mathrm{Mg}, 5$ de $\mathrm{Zn}$ e 2 de B. Nos tratamentos de gotejamento, $85 \%$ do $\mathrm{N}$ e do $\mathrm{K}$ e $30 \%$ do Ca foram aplicados via água de irrigação ao longo do ciclo de desenvolvimento da cultura. No tratamento TA, $65 \%$ do N, $40 \%$ do $\mathrm{K}$ e $30 \%$ do $\mathrm{Ca}$ foram aplicados em cobertura aos 25 e 50 dias após o transplante de mudas. Os demais nutrientes e o restante do $\mathrm{N}$, do $\mathrm{K}$ e do Ca foram aplicados por ocasião do transplante de mudas.

Nos primeiros 14 dias após o transplante todos os tratamentos foram irrigados por aspersão a cada 1-2 dias, uma vez que o pegamento de mudas no gotejamento subterrâneo, com laterais enterradas à profundidade igual ou superior a $20 \mathrm{~cm}$, é problemático.

Foi utilizada uma linha lateral de gotejadores, de fluxo turbulento, vazão de 4,0 L.h. ${ }^{-1} \cdot \mathrm{m}^{-1}$ a $90 \mathrm{kPa}$ e espaçados de $30 \mathrm{~cm}$, por linha de plantas. As irrigações foram realizadas a cada 3-4 dias até o início da frutificação, a cada 2 dias na fase de desenvolvimento de frutos e a cada 3-4 dias após 10\% de frutos maduros (Marouelli \& Silva, 2000). Paralisou-se as irrigações quando as plantas apresentaram cerca de $60 \%$ de frutos maduros.

A quantidade de água aplicada por irrigação foi igual para todos os trata-

\footnotetext{
${ }^{1}$ Comunicação pessoal do Eng. Agr. Rafael de Rezende Sant'Ana, da Unilever BestFoods, Goiânia (GO), em 26.09.2001.
} 
Tabela 1. Média da tensão máxima de água do solo $(\mathrm{kPa})$ por ocasião das irrigações nos estádios de desenvolvimento do tomateiro e profundidades do solo, nos diferentes tratamentos de irrigação. Brasília, Embrapa Hortaliças, 2000.

\begin{tabular}{|c|c|c|c|c|c|c|c|c|c|c|c|c|}
\hline \multirow{4}{*}{$\begin{array}{c}\text { Estádio } \\
\text { (dias pós transplante) }\end{array}$} & \multicolumn{12}{|c|}{ Tratamento } \\
\hline & \multicolumn{3}{|c|}{ TGS } & \multicolumn{3}{|c|}{ TG20 } & \multicolumn{3}{|c|}{ TG40 } & \multicolumn{3}{|c|}{ TA } \\
\hline & \multicolumn{12}{|c|}{ Profundidade do solo $(\mathrm{cm})$} \\
\hline & 10 & 30 & 50 & 10 & 30 & 50 & 10 & 30 & 50 & 10 & 30 & 50 \\
\hline Pegamento de mudas (1-14) & 12 & 18 & 27 & 12 & 18 & 27 & 12 & 18 & 27 & 12 & 18 & 27 \\
\hline Vegetativo (15-30) & 37 & 28 & 19 & 44 & 36 & 17 & 58 & 32 & 18 & 170 & 58 & 34 \\
\hline Frutificação (31-90) & 18 & 20 & 29 & 48 & 42 & 20 & 220 & 45 & 12 & 45 & 35 & 29 \\
\hline Maturação 1 (91-103) & 64 & 57 & 40 & 64 & 52 & 29 & 195 & 62 & 23 & 120 & 60 & 46 \\
\hline Maturação 2 (104-115) & 4 & 9 & 30 & 5 & 12 & 20 & 5 & 15 & 22 & 5 & 10 & 25 \\
\hline
\end{tabular}

Obs.: TGS - gotejo superficial; TG20 - gotejo a 20 cm; TG40 - gotejo a 40 cm; TA - aspersão; Maturação 1 - antes das chuvas; Maturação $2-$ após as chuvas.

mentos por gotejamento, sendo a lâmina necessária para repor a evapotranspiração da cultura, computada conforme Keller \& Bliesner (1990), a partir de dados diários da evaporação do tanque Classe A, coeficientes de cultura reportados por Allen et al. (1998) e fração de área sombreada. O manejo de água no tratamento TA foi realizado segundo Marouelli \& Silva (2000), utilizando-se o tanque Classe A.

Tensiômetros foram instalados nas profundidades de $10 ; 30$ e $50 \mathrm{~cm}$ para monitorar a tensão de água no perfil do solo. Avaliações da umidade do solo, pelo método gravimétrico, foram realizadas para as condições fora do limite de funcionamento do tensiômetro, o que ocorreu no tratamento TA e para a profundidade de $10 \mathrm{~cm}$ do tratamento TG40.

Foram avaliados o estande inicial e final, profundidade efetiva do sistema radicular, massa seca da parte aérea (excluindo os frutos), produtividade comercial, massa média de fruto, número de frutos comerciais por planta, frutos podres, com podridão apical e verdes, sólidos solúveis totais e eficiência do uso de água (EA), em quilogramas de frutos comerciais por metro cúbico de água total aplicada. Os dados foram submetidos à análise de variância, empregando-se o teste "F", sendo as médias das variáveis afetadas significativamente pelos tratamentos comparadas pelo teste de Duncan.

A profundidade radicular efetiva, definida como sendo correspondente a $80-85 \%$ da quantidade de raízes acumu- ladas ao longo do perfil do solo, foi determinada pelo método do perfil reticulado do solo (Atkinson \& MackieDawson, 1991) em duas das seis repetições de cada tratamento. Utilizou-se uma malha de $5 \mathrm{~cm} \times 5 \mathrm{~cm}$, até uma profundidade de $110 \mathrm{~cm}$, onde se contou o número de interseções das raízes com as linhas da malha para então estimar o comprimento total das raízes dentro de cada malha.

\section{RESULTADOS E DISCUSSÃO}

A lâmina bruta total de água aplicada foi de $370 \mathrm{~mm}$ (50 irrigações) nos tratamentos irrigados por gotejamento e de $430 \mathrm{~mm}$ (28 irrigações) no irrigado por aspersão. A precipitação pluvial durante os primeiros 101 dias após o transplante de mudas foi de $0,3 \mathrm{~mm}$, enquanto que a precipitação nos 14 dias que antecederam a colheita totalizou $148,6 \mathrm{~mm}$.

Os valores médios das tensões máximas de água do solo antes de cada irrigação, nos diferentes estádios de desenvolvimento do tomateiro, nas profundidades de 10; 30 e 50 cm são apresentadas na Tabela 1. Muito embora a freqüência e a lâmina de irrigação tenham sido iguais para todos os tratamentos por gotejamento, as plantas foram submetidas a diferentes níveis de estresse hídrico. A tensão máxima de água do solo, estimada a $50 \%$ da profundidade efetiva das raízes, foi em média igual a $25 \mathrm{kPa}$ para o tratamento TGS, $40 \mathrm{kPa}$ para o TG20, $80 \mathrm{kPa}$ para o TG40 e 55 kPa para o TA.
$\mathrm{O}$ ciclo vegetativo das plantas não foi afetado significativamente $(p>0,05)$ pelos tratamentos irrigados por gotejamento e por aspersão. A colheita de todas parcelas foi realizada aos 115 dias após o transplante de mudas e a concentração média de maturação de frutos foi de $95,2 \%$ (em massa).

A profundidade efetiva do sistema radicular da cultura, observada no final do ciclo de desenvolvimento das plantas, foi de $40 \mathrm{~cm}$ para o tratamento TGS, de $48 \mathrm{~cm}$ para o TG20, de $57 \mathrm{~cm}$ para o TG40 e de $45 \mathrm{~cm}$ para o TA. O maior crescimento das raízes ocorreu nas maiores profundidades de instalação dos gotejadores, em razão do fornecimento de água e, principalmente, de nutrientes nas camadas mais profundas do solo.

A irrigação por aspersão, durante os primeiros quatorze dias após o transplante, garantiu um pegamento de mudas uniforme, com média de 27.585 plantas.ha ${ }^{-1}$ aos 21 dias após o transplante. $\mathrm{O}$ estande final (média de 27.501 plantas.ha-1) e a massa média de fruto comercial (média de 78,8 g) não foram afetadas $(\mathrm{p}>0,05)$ pelos tratamentos. $\mathrm{O}$ efeito não significativo dos tratamentos sobre o estande final indica que a lâmina total de água disponível de $56 \mathrm{~mm}$, na camada de 0 a $40 \mathrm{~cm}$ do solo, por ocasião do início dos tratamentos, foi suficiente para que as raízes das plantas do tratamento TG20 e, principalmente, do TG40 se desenvolvessem até as camadas do solo onde a água foi fornecida.

A maior massa seca da parte aérea foi verificada para os tratamentos TA e TGS, enquanto a menor massa ocorreu 
Tabela 2. Massa seca da parte aérea (MS), produtividade comercial (PC), número de frutos comerciais por planta (NF), porcentagem, em número, de frutos podres (FP) e eficiência de uso de água (EA) do tomateiro industrial para os tratamentos de irrigação. Brasília, Embrapa Hortaliças, 2000.

\begin{tabular}{lccccc}
\hline \multicolumn{1}{c}{ Tratamento } & MS $\left(\mathbf{t ~ h a ~}^{-1}\right)$ & PC $\left(\mathbf{t ~ h a} \mathbf{~}^{-1}\right)$ & EA $\left(\mathbf{k g} \cdot \mathbf{m}^{-3}\right)$ & NF & FP (\%) \\
\hline Gotejo superficial & $13,8 \mathrm{ab}$ & $124 \mathrm{a}$ & $33,5 \mathrm{a}$ & $54,9 \mathrm{a}$ & $7,3 \mathrm{~b}$ \\
Gotejo $20 \mathrm{~cm}$ & $13,3 \mathrm{~b}$ & $116 \mathrm{ab}$ & $31,4 \mathrm{a}$ & $53,8 \mathrm{a}$ & $2,7 \mathrm{c}$ \\
Gotejo $40 \mathrm{~cm}$ & $11,0 \mathrm{c}$ & $94 \mathrm{c}$ & $25,4 \mathrm{~b}$ & $44,4 \mathrm{~b}$ & $2,8 \mathrm{c}$ \\
Aspersão & $15,3 \mathrm{a}$ & $108 \mathrm{~b}$ & $25,1 \mathrm{~b}$ & $51,3 \mathrm{ab}$ & $15,5 \mathrm{a}$ \\
\hline
\end{tabular}

Obs.: Médias seguidas pela mesma letra, nas colunas, não diferem entre si (p>0,05), pelo teste de Duncan.

para o TG40, sendo que as massas nos tratamentos TGS e TG20 não diferiram entre si (Tabela 2). O menor crescimento vegetativo de plantas no tratamento TG20 e, especialmente no TG40, resultou da maior deficiência de água no solo a que as plantas foram submetidas, especialmente nos estádios vegetativo e de frutificação (Tabela 1). Marouelli et al. (1991) verificaram redução da massa seca da parte aérea de 3,0 kg.ha ${ }^{1} \cdot \mathrm{kPa}^{-1}$ e de 2,0 kg.ha-1 $\mathrm{kPa}^{-1}$ com o aumento da tensão de água do solo nos estádios vegetativo e de frutificação, respectivamente, de tomateiro irrigado por aspersão.

A maior produtividade comercial de frutos (124 t.ha-1) ocorreu no tratamento TGS, a qual não diferiu estatisticamente do TG20 (Tabela 2). Por sua vez, não houve diferença de produtividade entre os tratamentos TG20 e TA. A produtividade no tratamento TG40 foi estatisticamente menor entre todos os tratamentos, resultante da maior deficiência de água no solo que as plantas foram submetidas (Tabela 1). Segundo Marouelli et al. (1991), a ocorrência de deficiência hídrica, principalmente durante o estádio de frutificação, reduz sobremaneira a produtividade do tomateiro. A menor produtividade no tratamento TA, comparativamente aos tratamentos TGS e TG20, foi devido à maior tensão de água do solo que as plantas foram submetidas (Tabela 1) e ao fato da aspersão favorecer maior incidência de doenças foliares e apodrecimento de frutos (Marouelli \& Silva, 2000). Resultados similares foram obtidos por Prieto et al. (1999), os quais verificaram que a produtividade na irrigação por gotejamento foi cerca de $20 \%$ maior que na aspersão.
A maior EA ocorreu nos tratamentos TGS e TG20 (média de 32,5 kg.m ${ }^{-3}$ ), enquanto que a menor foi obtida nos tratamentos TA e TG40 (Tabela 2). A menor EA do tratamento TA, comparativamente aos tratamentos TGS e TG20, deveu-se provavelmente às seguintes razões: a menor uniformidade de aplicação de água do sistema por aspersão; e a aspersão molhar a parte aérea das plantas favorecendo doenças. A menor EA do tratamento TG40 pode ser atribuída à deficiência de água a que as plantas foram submetidas e à drenagem de parte da água aplicada abaixo do sistema radicular das plantas (Tabela 1). Os valores de EA dos tratamentos TGS e TG20 são semelhantes ao máximo obtido por Phene et al. (1992) de 31 $\mathrm{kg} . \mathrm{m}^{-3}$ e cerca de $60 \%$ maior ao relatado por Prieto et al. (1999).

Os tratamentos afetaram o número de frutos comerciais por planta de forma similar à produtividade (Tabela 2), ou seja, maior número de frutos ocorreu nos tratamentos TGS e TG20 e menor no tratamento TG40. Assim, conforme também observado por Prieto et al. (1999), a produtividade esteve diretamente correlacionada ao número de frutos por planta, uma vez que o estande final e a massa média de frutos não foram afetadas pelos tratamentos.

A porcentagem de frutos podres no tratamento TA foi $112 \%$ maior que no tratamento TGS e $453 \%$ maior que nos tratamentos TG20 e TG40 (Tabela 2). O maior apodrecimento de frutos devese em razão da aspersão molhar freqüentemente a folhagem das plantas e dos frutos, favorecendo maior incidência de doenças (Marouelli \& Silva, 2000). A menor taxa de frutos podres nos tratamentos TG20 e TG40, em relação ao TGS, foi devido a superfície do solo e dossel vegetativo da cultura permanecerem praticamente secos, mesmo por ocasião das irrigações, o que contribuiu para minimizar a incidência de doenças (Silva et al., 1999; Lopes \& Santos, 1994). Por outro lado, constatou-se que, durante pelo menos $10 \%$ do ciclo da cultura, o dossel vegetativo no tratamento TGS, ao contrário dos TG20 e TG40, apresentavase molhado durante as primeiras horas do dia, certamente em razão da condensação da água evaporada da superfície do solo durante à noite, o que pode ter favorecido um maior apodrecimento de frutos. Não houve ocorrência de frutos com podridão apical em nenhum dos tratamentos.

O teor de sólidos solúveis totais (média de 4,2\%) não foi afetado pelos tratamentos. Os valores relativamente baixos de sólidos solúveis e o efeito não significativo dos tratamentos, mesmo quando a cultura foi submetida a níveis extremos de água no solo $(25$ a $80 \mathrm{kPa})$, foram ocasionados provavelmente pela ocorrência de chuvas nas últimas duas semanas que precederam a colheita dos frutos.

A profundidade de instalação da linha lateral de $20 \mathrm{~cm}$ foi suficiente para não provocar afloramento da umidade na superfície do solo e garantir produtividade estatisticamente igual à obtida via gotejamento superficial, mas insuficiente para que as operações convencionais de preparo de solo possam ser realizadas sem risco de dano às laterais. Assim, a profundidade de $20 \mathrm{~cm}$ somente seria viável se práticas alternativas de cultivo, como por exemplo plantio direto, possuem utilizadas tanto para o tomateiro quanto para as culturas em sistema de rotação.(Camp, 1998). 


\section{AGRADECIMENTOS}

À Rain Bird Brasil pela doação de parte dos sistemas de irrigação por gotejamento e por aspersão utilizados e à Unilever BestFoods pelo fornecimento das mudas de tomateiro.

\section{LITERATURA CITADA}

ALLEN, R.G.; PEREIRA, L.S.; RAES, D.; SMITH, M. Crop evapotranspiration: guidelines for computing crop water requirements. Roma: FAO, 1998. 328 p. (Irrigation and Drainage Papers, 56).

ATKINSON, D.; MACKIE-DAWSON, L.A. Roo growth: methods of measurement. In: SMITH, K.A.; MULLINS, C.E. Soil analysis. New York: Marcel Dekker, 1991. p. 447-509.

BARBOSA, V. The processing tomato growing system under tropical and subtropical conditions: the Brazilian experience. In: INTERNATIONAL CONFERENCE ON THE PROCESSING TOMATO, 1., 1996, Recife. Proceedings... Alexandria: American Society for Horticultural Science/Empresa Pernambucana de Pesquisa Agropecuária. 1997. p. 94-97
CAMP, C.R. Subsurface drip irrigation: a review. Transaction of the ASAE, v. 41, n. 5, p. 1353-1367, 1998.

CASAÑO, C. Informacion geral: riego por goteo subterráneo en olivar. Valencia: Mondragon, 1998. $7 \mathrm{p}$.

KELLER, J.; BLIESNER, R.D. Sprinkle and trickle irrigation. New York: VanNostrand Reinhold, 1990. 652 p.

LOPES, C.A.; SANTOS, J.R.M. Doenças do tomateiro. Brasília: EMBRAPA-SPI/EMBRAPACNPH, 1994. 67 p.

MAROUELLI, W.A.; SILVA, H.R.; OLIVEIRA C.A.S. Produção de tomate industrial sob diferentes regimes de umidade no solo. Pesquisa Agropecuária Brasileira, Brasília, v. 26, n. 9, p. 1531-1537, 1991.

MAROUELLI, W.A.; SILVA, W.L.C. Irrigação. In: SILVA, J.B.C.; GIORDANO, L.B., ed. Tomate para processamento industrial. Brasília: Embrapa, 2000. p.60-71.

MAROUELLI, W.A.; SILVA, W.L.C.; SILVA,

H.R. Seleção de sistemas de irrigação para o cultivo de tomateiro industrial. In: CONGRESSO BRASILEIRO DE ENGENHARIA AGRÍCOLA,

27., 1998, Poços de Caldas. Anais... Poços de Caldas: SBEA-UFLA, 1998. v. 2, p. 67-69.

MAY, D.M. Water management differences between drip- and furrow-irrigated processing tomatoes to maximize yield and fruit quality in California. In: INTERNATIONAL CONFERENCE ON THE PROCESSING TOMATO, 1., 1996, Recife. Proceedings... Alexandria: ASHS/IPA, 1997. p.54-58.
PHENE, C.J.; HUTMACHER, R.B.; DAVIS, K.R. Two hundred tons per hectare of processing tomatoes - Can we reach it?. HortTechnology, v. 2, n. 1, p. 16-22, 1992.

PRIETO, M.H.; LÓPEZ, J.; BALLESTEROS, R. Influence of irrigation system and strategy of the agronomic and quality parameters of the processing tomatoes in Extremadura. Acta Horticulturae, v. 487, p. 575-579, 1999.

SILVA, W.L.C.; GIORDANO, L.B.; MAROUELLI, W.A.; FONTES, R.R.; GORNAT, B. Response of six processing tomatoes cultivars to subsurface drip fertigation. Acta Horticulturae, n. 487, p. 569-573, 1999.

SILVA, W.L.C; MAROUELLI, W.A. Exploratory studies on microirrigation for processing tomatoes in Central Brasil. In: INTERNATIONAL MICROIRRIGATION CONGRESS, 5., 1995, Orlando. Proceedings... Orlando: ASAE, 1995. p. 904-908.

VAN GENUCHTEN, M.T. A closed-form equation for predicting the hydraulic conductivity of unsaturated soils. Soil Science Society American Journal, v. 50, p. 288-291, 1980. 